\title{
Avaliando Valores a partir de Relatos Comportamentais: Evidências Psicométricas de uma Nova Medida
}

\author{
Tiago Jessé Souza Lima \\ Valdiney Veloso Gouveia \\ Luana Elayne Cunha Souza \\ Patrícia Nunes Fonseca \\ Universidade Federal da Paraíba \\ Paraíba, PB, Brasil
}

\begin{abstract}
RESUMO
Este estudo teve por objetivo reunir evidências de validade fatorial e consistência interna de um novo instrumento de valores, partindo da teoria funcionalista dos valores. Seus itens expressam os valores como comportamentos autoinformados, sendo menos abstrato do que instrumentos prévios. Participaram deste estudo 388 indivíduos com idades variando de 14 a 25 anos $(M=19,0 ; D P=2,50)$, regularmente matriculados em instituições públicas de ensino. Eles responderam a medida de valores com 54 itens e perguntas demográficas. Os resultados apoiaram empiricamente a medida proposta, que permitiu corroborar as hipóteses de conteúdo (valores agrupados em seis subfunções) e estrutura (valores organizados em torno de dois eixos, orientação e motivação) dessa teoria. Concluiu-se que o instrumento ora descrito se mostrou psicometricamente adequado, podendo ser útil para medir os valores em pessoas com menor nível educacional, que são favorecidas ao lidar com itens contextualizados, que demandam menos abstração ou capacidade cognitiva.
\end{abstract}

Palavras-chave: Medida; Valores; Comportamentos; Validade; Precisão.

\section{ABSTRACT}

Assessing Values from Behavioral Reports: Psychometric Evidences of a New Measure

This study aimed to gather evidence of factorial validity and internal consistency of a new instrument to measure values, taking into account the functional theory of values. Its items express the values as self-reported behaviors, being less abstract than previous instruments. The study included 388 individuals aged 14-25 years (mean=19,0 $\mathrm{SD}=2.5$ ), regularly enrolled in public education. They answered the measure of values composed by 54 items and demographic questions. Results provided empirical support for the proposed measure, which permitted to corroborate the content (values grouped in six sub-functions) and structure (values organized around two axes, orientation and motivator) hypotheses based on this theory. In conclusion, the instrument described presented itself as adequate, being useful to measure values in people with low educational level, who are beneficed to deal with contextualized items, which demand less abstraction and cognitive capacity.

Keywords: Measure; Values; Behaviors; Validity; Reliability.

\section{RESUMEN}

\section{Evaluando Valores de Informes de Comportamiento: Evidencias Psicométricas de una Nueva Medida}

El objetivo de este estudio ha sido reunir pruebas de validez factorial y fiabilidad de un instrumento nuevo para medir valores, partiendo de la teoría funcionalista de los valores. Sus ítems expresan valores como comportamientos autoinformados, siendo menos abstracto que los instrumentos anteriores. Participaron del estudio 388 personas de edades entre 14 y 25 años $(M=19.0, D T=2.50)$, regularmente matriculados en la educación pública. Éstos contestaron la medida de los valores y preguntas demográficas. Los resultados apoyaron empíricamente la medida propuesta, permitiendo corroborar las hipótesis de contenido (valores agrupados en seis sub-funciones) y estructura (valores se organizan en torno a dos ejes, orientación y motivador) de esta teoría. Se concluyó que el instrumento descrito se mostró psicométricamente adecuado, pudiendo ser útil para medir los valores en personas con menor nivel de estudios, que son favorecidos al tratar con ítems contextualizados, que requieren menos abstracción o capacidad cognitiva.

Palabras clave: Medición; Valores; Comportamientos; Validez; Fiabilidad. 
Desde as contribuições de Thomas e Znaniecki (1918), passando por Parsons e Shils (1951), Kluckhohn (1951), Maslow (1954) e Rokeach (1973), o estudo dos valores humanos acumulou conhecimentos evidentes (Maio, 2010; Ros \& Gouveia, 2006). Embora o campo teórico tenha avançado, foram escassos os estudos empíricos levados a cabo por estes pesquisadores até os anos 1970, principalmente aqueles destinados a testar a validade transcultural dos modelos apresentados. Esta lacuna viria a ser preenchida pelos estudos desenvolvidos por Schwartz (Schwartz, 1992), que propôs um modelo sobre o conteúdo e a organização dos valores. Embora o modelo de Schwartz tenha como base propostas teóricas anteriores, como a de Rokeach, apresenta limitação ao se pautar predominantemente em dados empíricos, razão que pode explicar o fato de ter sofrido repetidas alterações ao longo do tempo, variando de sete a dezenove tipos motivacionais, embora o modelo com dez tipo seja o mais conhecido (Schwartz \& Boehnke, 2004; Schwartz et al., 2012).

Neste cenário, na última década foi sendo delineada uma teoria diferente acerca dos valores humanos, que possivelmente supre algumas das limitações do modelo de Schwartz. Dita teoria foi originalmente proposta por Gouveia (2003; Gouveia, Milfont, Fischer, \& Santos, 2008), reunindo evidências empíricas satisfatórios em diferentes países e continentes (Ardila, Gouveia, \& Medeiros, 2012; Gouveia, 2013; Medeiros et al., 2012). Em suma, esta teoria funcionalista dos valores foi proposta como uma alternativa aos modelos prévios, não pretendendo suplantá-los (Fischer, Milfont, \& Gouveia, 2011; Gouveia, Fonseca, Milfont, \& Fischer, 2011). Trata-se de uma contribuição, uma forma sistemática, parcimoniosa e integradora de pensar acerca dos valores humanos, sendo construída na direção do estabelecido a partir da teoria da ação (Kluckhohn, 1951; Parsons \& Shils, 1951), passando pela abordagem mais psicológica (Maslow, 1954; Rokeach, 1973) e encontrando respaldo em modelos mais recentes (Inglehart, 1989; Schwartz, 1992).

$\mathrm{O}$ instrumento comumente empregado para medir os valores de acordo com esta teoria é o Questionário dos Valores Básicos (QVB), contando com 18 itens (Gouveia et al., 2008). Embora o QVB tenha mostrado evidências satisfatórias de validade e precisão, alguns problemas podem ser apontados. Primeiro, trata-se de uma medida que requer um nível de pensamento abstrato por apresentar os valores fora de contextos específicos. Desta forma, requer maior nível de abstração dos respondentes, o que dificulta sua administração em amostras com nível educacional mais baixo. Além disso, seus itens são caracterizados por duas afirmações cada (e.g., Obediência. Cumprir seus deveres e obrigações do dia a dia; respeitar seus pais, os superiores e os mais velhos), o que pode gerar confusão e dificultar a resposta. De forma similar, Schwartz et al. (2001) apontam dificuldades parecidas no Schwartz Values Survey (SVS), propondo um novo instrumento (o Portrait Values Questionnaire, PVQ) com o intuito de superar o caráter abstrato dos itens, que tem sido mais empregado nos desenvolvimentos recentes desta teoria (Schwartz et al., 2012).

A partir destas observações, o presente artigo tem como objetivo propor um instrumento novo de medição dos valores, partindo da teoria funcionalista dos valores. Neste caso, tendo em conta este modelo teórico, elaborou-se um instrumento com itens que expressavam valores de forma mais concreta, a partir de relatos comportamentais, provendo assim uma medida que requer menos abstração e esforço cognitivo por parte do respondente.

Posteriormente, procurou-se conhecer evidências de validade de construto desta medida, tendo em conta as hipóteses de conteúdo (seis subfunções) e estrutura (organização das subfunções de acordo com os tipos de orientação e motivador) da teoria. Além disso, checaram-se evidências de seus parâmetros de consistência interna (alfa de Cronbach, homogeneidade e confiabilidade composta).

\section{TEORIA FUNCIONALISTA DOS VALORES HUMANOS}

Na teoria funcionalista os valores são formalmente definidos como (1) critérios de orientação que guiam as ações humanas e (2) expressam cognitivamente suas necessidades básicas. Assim, seu foco principal está nas funções dos valores, que são definidas como os aspectos psicológicos que estes cumprem ao guiar comportamentos e representar cognitivamente as necessidades humanas (Gouveia, 2013; Gouveia, Fonseca et al., 2011). Os autores propõem duas funções: a primeira diz respeito aos valores como guias das ações do homem, sendo denominada como tipo de orientação (Rokeach, 1973; Schwartz, 1992); a segunda remete aos valores como expressão das necessidades humanas, nomeada como tipo de motivador (Inglehart, 1977; Maslow, 1954).

$\mathrm{Na}$ primeira função, os valores guiam os comportamentos dos indivíduos em uma orientação social (o indivíduo na comunidade) ou pessoal (o indivíduo por si mesmo). O comportamento orientado pelos valores sociais é centrado na sociedade ou possuem um foco interpessoal, enquanto aqueles guiados por valores pessoais são egocêntricos ou possuem um foco intrapessoal (Rokeach, 1973). Assim, os indivíduos tendem a enfatizar ou o grupo (valores 
sociais) ou a eles mesmos (valores pessoais) como a unidade principal de sobrevivência (Gouveia, Milfont, Fischer, \& Coelho, 2009). Além destes, há um terceiro grupo de valores que se situam teoricamente entre os sociais e os pessoais, denominados de centrais (o propósito geral da vida). A centralidade destes valores não é situacional, isto é, não se deve ao juízo que as pessoas fazem acerca de sua importância (Verplaken \& Holland, 2002); é, por outro lado, estrutural. Destacase que estes valores são a base a partir da qual são definidos os demais, representando cognitivamente a polarização de necessidades mais básicas (e.g., comer, dormir, beber) e aquelas de ordem mais elevada (e.g., cognitivas, estéticas, de autorrealização). Deste modo, a primeira função dos valores como guia dos comportamentos humanos é identificada pela dimensão funcional denominada como tipo de orientação, com três possibilidades: social, central e pessoal.

$\mathrm{Na}$ segunda função, os valores expressam as necessidades humanas como um motivador, que pode ser materialista (a vida como fonte de ameaças) ou idealista (a vida como fonte de oportunidades) (Inglehart, 1977; Ronen, 1994). Valores materialistas indicam ideias práticas, sugerindo uma orientação para metas específicas, visando um interesse imediato e a observância de regras normativas. Por sua vez, valores idealistas expressam uma orientação universal, com ideias e princípios mais abstratos, não sendo necessariamente dirigidos a metas concretas e, geralmente, são não específicos. Esta segunda função dos valores, de dar expressão cognitiva às necessidades humanas, é identificada pela dimensão funcional denominada como tipo de motivador, representada por valores materialistas (pragmáticos) ou idealistas (humanitários; Gouveia et al., 2009). Estas duas dimensões (tipo de orientação e tipo de motivador) formam dois eixos na representação espacial da estrutura dos valores, conforme pode ser observado na Figura 1.
A primeira dimensão, eixo horizontal, corresponde ao tipo de orientação (pessoal, central e social), enquanto o eixo vertical corresponde ao tipo de motivador (materialista e idealista). $\mathrm{O}$ cruzamento destas duas funções permite identificar a estrutura $3 \times 2$ mostrada na Figura 1, onde são apresentadas as seis subfunções derivadas dos valores: experimentação, realização, existência, suprapessoal, interativa e normativa. As setas que emanam das subfunções existência e suprapessoal indicam que os valores que as representam são a fonte principal ou a referência dos outros valores (Gouveia, Fonseca et al., 2011).

A subfunção existência representa as necessidades fisiológicas mais básicas (e.g., comer, beber, sobrevivência) e de segurança (Maslow, 1954; Ronen, 1994). Seu propósito principal é assegurar as condições básicas para a sobrevivência biológica e psicológica do indivíduo. Possuem uma orientação central e um motivador materialista, sendo a fonte das outras duas subfunções: realização e normativa (Gouveia et al., 2009). A subfunção realização representa as necessidades de autoestima (Maslow, 1954; Ronen, 1994), correspondendo a uma orientação pessoal e um motivador materialista. Tais valores se originam de um princípio pessoal para guiar a vida dos indivíduos, enquanto focaliza realizações materiais; a realização pode ser uma exigência para interações sociais prósperas e para o funcionamento institucional adequado (Schwartz, 1992). A terceira subfunção é a normativa, cujo motivador é materialista, mas com orientação social. A necessidade de controle e as precondições imprescindíveis para alcançar todas as necessidades, isto é, demandas institucionais e sociais (Maslow, 1954; Schwartz, 1992), são cognitivamente representadas por valores desta subfunção. Reflete, portanto, a importância de preservar a cultura e as normas convencionais (Gouveia et al., 2009).

\begin{tabular}{|c|c|c|c|c|}
\hline \multirow{4}{*}{ 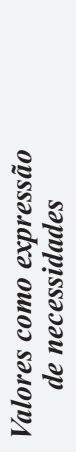 } & & \multicolumn{3}{|c|}{ Valores como padrão-guia de comportamentos } \\
\hline & & $\begin{array}{c}\text { Metas pessoais } \\
\text { (o indivíduo por si mesmo) }\end{array}$ & $\begin{array}{c}\text { Metas centrais } \\
\text { (o propósito geral da vida) }\end{array}$ & $\begin{array}{c}\text { Metas sociais } \\
\text { (o indivíduo na comunidade) }\end{array}$ \\
\hline & $\begin{array}{l}\text { Necessidades idealistas } \\
\text { (a vida como fonte de oportunidades) }\end{array}$ & $\begin{array}{c}\text { Experimentação } \\
\text { Emoção } \\
\text { Prazer } \\
\text { Sexualidade }\end{array}$ & $\begin{array}{l}\text { Suprapessoal } \\
\text { Beleza } \\
\text { Conhecimento } \\
\text { Maturidade }\end{array}$ & $\begin{array}{l}\text { Interativa } \\
\text { Afetividade } \\
\text { Apoio social } \\
\text { Convivência }\end{array}$ \\
\hline & $\begin{array}{l}\text { Necessidades materialistas } \\
\text { (a vida como fonte de ameaças) }\end{array}$ & $\begin{array}{l}\text { Realização } \\
\text { Êxito } \\
\text { Poder } \\
\text { Prestígio }\end{array}$ & $\begin{array}{c}\text { Existência } \\
\text { Estabilidade } \\
\text { Saúde } \\
\text { Sobrevivência }\end{array}$ & $\begin{array}{c}\text { Normativa } \\
\text { Obediência } \\
\text { Religiosidade } \\
\text { Tradição }\end{array}$ \\
\hline
\end{tabular}

Figura 1. Funções, subfunções e valores específicos. 
Os valores da subfunção suprapessoal apresentam orientação central e motivador idealista. Seus valores representam as necessidades estéticas, de cognição e autorrealização (Maslow, 1954). Estes valores ajudam a estruturar o mundo de uma forma consistente, fornecendo claridade e estabilidade na organização cognitiva da pessoa, indicando a importância de ideias abstratas, com menor ênfase em coisas concretas e materiais (Inglehart, 1977). É a fonte das subfunções de mesmo motivador: experimentação e interativa. A subfunção experimentação é composta por um motivador idealista com orientação pessoal, representando a necessidade fisiológica de satisfação, em sentido amplo, ou o princípio do prazer (hedonismo; Maslow, 1954; Ronen, 1994). Esta subfunção é menos pragmática na busca de alcançar status social ou assegurar harmonia e segurança sociais; seus valores contribuem para a promoção de mudanças e inovações na estrutura das organizações sociais. Por fim, a subfunção interativa apresenta um motivador idealista com uma orientação social, acentuando o destino comum e a experiência afetiva entre os indivíduos. Representa as necessidades de pertencimento, amor e afiliação (Maslow, 1954). Seus valores são essenciais para estabelecer, regular e manter as relações interpessoais.

A representação adequada dessas seis subfunções valorativas por meio de indicadores ou valores específicos diz respeito à hipótese de conteúdo dos valores. Deste modo, a validade do conteúdo dos valores não está ligada a um conjunto de valores específicos, mas sim à representação adequada das seis subfunções, sendo possível que outros pesquisadores considerem, de acordo com sua cultura ou contexto de pesquisa, um conjunto diferente de valores. A teoria também prevê uma estrutura que identifica duas funções principais que, combinadas, dão origem a seis subfunções valorativas, consonante com a Figura 1. Em termos pragmáticos, espera-se que seja possível identificar as seis subfunções valorativas, representadas por seus valores específicos.

A estrutura dos valores indica que estes podem ser representados em um espaço bidimensional formado pelos eixos horizontal (tipo de orientação) e vertical (tipo de motivador). No primeiro eixo, prevê-se que os valores centrais se localizem no centro do espaço, com os de orientação pessoal de um lado e, do outro, aqueles de orientação social. No segundo eixo, prevê-se que os tipos de motivadores materialista e idealista se localizem em regiões diferentes deste espaço. Portanto, a estrutura dos valores se refere à representação espacial das seis subfunções valorativas resultantes do cruzamento (combinação) das duas dimensões funcionais anteriormente descritas (hipótese de estrutura).
O conteúdo e a estrutura dos valores, conforme definidos pela teoria funcionalista, têm sido confirmados em amostras transculturais de estudantes e não estudantes de diversos países (Ardila et al., 2012; Gouveia, 2013; Gouveia et al., 2010). De acordo com seus autores, esta teoria apresenta vantagens frente a outras previamente propostas, como as que se indicam (Gouveia et al., 2009): (1) Parcimônia. Partindo unicamente de duas dimensões funcionais dos valores, identifica seis subfunções representadas cada uma por três valores específicos; e (2) Integração. Permite englobar dois dos principais modelos teóricos da literatura, os quais acentuam os tipos de motivadores (Inglehart, 1989) e os tipos de orientação (Schwartz, 1992).

Em resumo, a teoria funcionalista dos valores parece adequada, reunindo evidências empíricas que dão provas de que é satisfatoriamente operacionalizada por meio do Questionário dos Valores Básicos (Gouveia, 2003). Entretanto, por sua característica mais geral e abstrata, estando ainda constituída por um número reduzido de itens por subfunção, esta medida, inclusive funcionando com crianças (Gouveia, Milfont, Soares, Andrade, \& Leite, 2011), tende a reunir indicadores de consistência interna limítrofes, como costuma acontecer com outras medidas de valores (Gouveia, 2013). Neste sentido, pensou-se realizar o presente estudo, tendo em conta dois objetivos principais: (1) propor um novo instrumento de mensuração menos abstrato pautado na teoria funcionalista dos valores (baseado em autorrelatos de comportamentos expressivos de valores) e testar sua validade de construto e consistência interna; e, considerando dados empíricos produzidos a respeito, (2) testar as hipóteses de conteúdo e estrutura desta teoria.

\section{MÉTODO}

\section{Participantes}

Participaram deste estudo 388 indivíduos com idades variando de 14 a 25 anos, apresentando média de 19 anos $(D P=2,50)$, todos regulamente matriculados em instituições públicas de ensino de João Pessoa (PB). A maioria deles era do sexo feminino (55\%). Tratou-se de amostra de conveniência, tendo participado as pessoas que, convidadas, concordaram em participar voluntariamente.

\section{Instrumentos}

Os participantes responderam informações demográficas (idade e sexo), disponibilizados no final do instrumento, e o Questionário dos Valores Básicos - Relatos Comportamentais (QVB-RC). Este foi elaborado com o fim de medir relatos comportamen- 
tais que expressem valores tendo como base a teoria de Gouveia (2003). Baseando-se no Questionário dos Valores Básicos (QVB; Gouveia 2003), três especialistas no tema foram incumbidos de elaborar ou reescrever os itens do $Q V B$, procurando formular itens que representassem determinados valores por meio de enunciados expressos em forma de comportamento. No total, elaboraram-se ao menos cinco itens para cada um dos 18 valores deste instrumento, representando suas seis subfunções [e.g., Compartilha seus sentimentos com pessoas próximas (afetividade/interativa), Participa de eventos religiosos (religiosidade/normativa), Procura ler e saber de tudo um pouco (conhecimento/suprapessoal), Faz valer a sua opinião ao se tomar uma decisão em grupo (poder/realização), Realiza atividades que envolvam risco elou perigo (emoção/experimentação) e Evita fazer dividas (estabilidade pessoal/existência)]. Com base no julgamento de dez especialistas, os itens inadequados, redundantes ou que expressavam mais de um valor foram excluídos. No caso, mantiveram-se aqueles que obtiveram concordância igual ou superior a 70\% entre os juízes. Posteriormente, foi realizada uma validação semântica com um grupo de dez estudantes do ensino fundamental para verificar a compreensão dos itens e formato da escala de resposta. A versão deste instrumento foi composta por 54 itens, sendo retidos nove itens por subfunção (três para cada valor). Os participantes eram solicitados a indicar com que frequência costumavam apresentar cada um dos comportamentos descritos, empregando escala de resposta de 7 pontos, variando de 0 (Nunca) a 6 (Sempre).

\section{Procedimento}

Os questionários foram aplicados coletivamente em sala de aula, porém respondidos individualmente. A coleta foi realizada por colaboradores devidamente treinados, instruídos a interferir o mínimo possível na aplicação, dando apenas explicações sobre como responder o questionário, evitando emitir comentários ou dar respostas quanto aos conteúdos abordados. Enfatizou-se o caráter voluntário da participação na pesquisa, garantindo o anonimato das respostas dadas, bem como o respeito às diretrizes éticas que regem a pesquisa com seres humanos (Resoluções 196/96, 215/97, 466/12 do Conselho Nacional de Saúde). No caso de pessoas de menor idade, solicitou-se a assinatura de termo de responsabilidade dos diretores das escolas; aqueles de maior idade tiveram que assinar termo de consentimento livre e esclarecido. Em média, 15 minutos foram suficientes para concluir a participação no estudo. O presente trabalho foi aprovado pelo comitê de ética do Hospital Universitário Lauro Vanderley (HU;UFPB; Protocolo 146/11).

\section{Análise dos dados}

Por meio do AMOS (versão 18) foram realizadas análises fatoriais confirmatórias (AFC) com o fim de conhecer evidências de validade fatorial da medida proposta. O método de estimação empregado foi o ML (Maximum Likelihood). Para testar o ajuste geral da solução fatorial, utilizaram-se quatro indicadores: a razão do qui-quadrado pelos graus de liberdade $\left(\chi^{2} / \mathrm{gl}\right)$, onde valores na faixa de 2-3 indicam uma ajuste adequado, admitindo-se até 5; o goodness of fit index (GFI) e o comparative fit index (CFI), onde valores iguais ou superiores a 0,90 indicam ajuste bom; e a root mean square error of approximation (RMSEA), cujos valores de 0,06 ou menor indicam ajuste satisfatório, aceitando-se aqueles de até 0,10 (Byrne, 2010). Além destes, a diferença de qui-quadrado $\left(\Delta \chi^{2}\right)$ e o expected cross-validation index (ECVI) foram empregados para avaliar melhoras entre os modelos concorrentes testados. Valores significativos de $\Delta \chi^{2}$ e valores mais baixos de ECVI refletem um modelo com melhor ajuste. O primeiro indicador é usado para comparar modelos aninhados e o segundo é útil para a comparação de modelos não-aninhados (Garson, 2012). Na AFC, além dos itens individuais, foram também empregados indicadores formados através de parcelas de itens. Este é um procedimento comumente utilizado nas técnicas de análise de variáveis latentes, que consiste no uso de indicadores agregados que são compostos pela soma ou média de dois ou mais itens, respostas ou comportamentos (Little, Cunningham, Shahar, \& Widaman, 2002).

O PASW (versão 18) foi utilizado para calcular os índices de consistência interna dos fatores e realizar análises de escalonamento multidimensional confirmatório (MDS - algoritmo PROXSCAL). A MDS confirmatória foi empregada para testar a adequação da estrutura proposta pela teoria, onde os valores se organizam em um espaço bidimensional, com o eixo horizontal correspondendo ao tipo de orientação, onde os valores centrais devem se localizar entre os pessoais e sociais; e o eixo vertical aos tipos de motivador materialista e idealista, que se localizam em regiões diferentes neste espaço. Para tanto, fixaram-se coordenadas para o tipo de orientação [experimentação $(1,0)$, realização $(1,0)$, existência $(0,0)$, suprapessoal $(0,0)$, interativa $(-1,0)$ e normativa $(-1,0)$ ] e o tipo de motivador [experimentação $(0,5)$, realização $(-0,5)$, existência $(-1,0)$, suprapessoal $(1,0)$, interativa $(0,5)$ e normativa $(-0,5)]$. Assumiu-se o nível ordinal de medida, permitindo break ties, sendo empregado o estimador Torgerson. O Phi de Tucker e o Raw Stress foram utilizados como indicadores de ajuste do modelo, aceitando-se valores 
acima de 0,90 para o primeiro e próximos à zero para o último (Borg, Groenen, \& Mair, 2012).

A consistência interna foi avaliada por meio do alfa de Cronbach, confiabilidade composta (CC) e índice de homogeneidade (correlação média interitens). Para os dois primeiro índices, valores iguais ou superiores a 0,70 asseguram a consistência interna da medida, embora valores na casa de 0,60 ou superiores serem aceitáveis; para o índice de homogeneidade são considerados valores iguais ou superiores a 0,20 como ideais, embora se admitam aqueles acima de 0,15 (Hair, Black, Babim, \& Anderson, 2009).

\section{RESULTADOS}

Inicialmente, realizaram-se análises fatoriais confirmatórias procurando testar três modelos. O primeiro modelo compreende as seis subfunções representadas por nove indicadores cada. $\mathrm{O}$ segundo considera os 18 valores específicos (usados no $Q V B$ ) como fatores de primeira ordem, com três itens para cada valor, e as subfunções como fatores de segunda ordem. Este modelo se justifica, pois se supõe que há uma variância compartilhada entre os itens que representam cada valor específico, que não é compartilhada pelos demais itens dos demais valores na mesma subfunção. Por fim, foi testado um modelo de parcela de itens no qual foram somados os itens que pertenciam a cada um dos 18 valores específicos, formando assim 18 parcelas compostas por três itens cada. Este modelo é presumivelmente mais indicado, pois possibilita melhor entendimento e aplicação mais prática da teoria, posto que é mais parcimonioso empregar indicadores compostos do que itens individualmente (para uma discussão mais aprofundada ver Little et al., 2002). Os índices de ajuste para os três modelos testados são apresentados na Tabela 1.

$\mathrm{O}$ modelo 1 apresentou os índices mais baixos. $\mathrm{O}$ segundo modelo apresentou índices melhores do que o primeiro, embora ainda não satisfatórios. Para ambos, todas as saturações foram estatisticamente diferentes de zero $(\lambda \neq 0 ; z>1,96, p<0,05)$. Como os modelos 1 e 2 apresentam igual número de variáveis observáveis, o teste de $\Delta \chi^{2}$ foi realizado. Os resultados apontaram que o modelo 2 apresentou uma melhora significativa no ajuste aos dados em comparação com o $1\left[\Delta \chi^{2}(27)=820,20 ; p<0,001\right]$. A solução que apresentou os melhores índices foi o modelo 3, de parcela de itens. Após uma análise inicial dos resíduos e índices de modificação (IM) deste modelo, procedeu-se a uma reespecificação, correlacionando os erros dos itens afetividade (e13) e apoio social (e14), em função de apresentarem um IM igual a 19 e por pertencerem a mesma subfunção. As cargas fatoriais variaram de 0,42 (tradição) a 0,76 (emoção), sendo todas estatisticamente diferentes de zero $(\lambda \neq 0 ; p<0,01)$. As covariâncias entre as subfunções foram significativas $(p<0,01)$, excetuando entre experimentação e existência. A estrutura fatorial do modelo $3 \mathrm{com}$ as respectivas cargas fatoriais para os 18 indicadores compostos (parcelas) são apresentados na Figura 2.

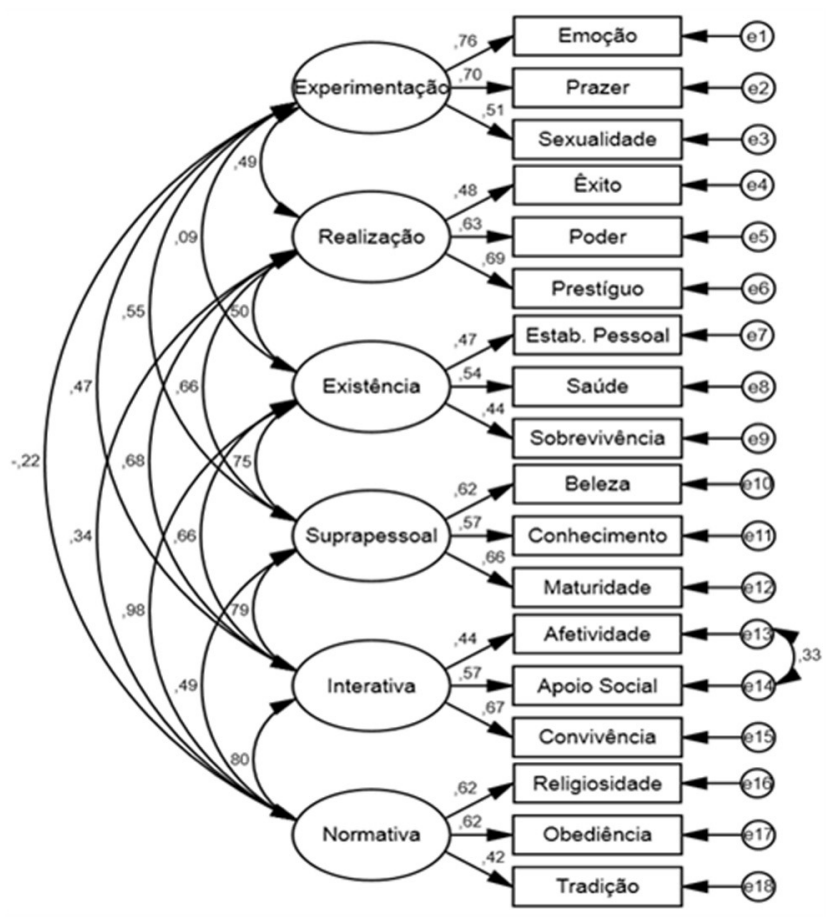

Figura 2. Estrutura fatorial do modelo e suas respectivas saturações.

TABELA 1

Índices de ajuste para os modelos alternativos testados.

\begin{tabular}{lccccccc}
\hline \multicolumn{1}{c}{ Modelo } & $\chi^{2}$ & $g l$ & $\chi^{2} / g l$ & GFI & CFI & RMSEA & ECVI \\
\hline Seis fatores & 3846,4 & 1362 & 2,82 & 0,70 & 0,58 & $\begin{array}{c}0,069 \\
(0,066-0,071)\end{array}$ & $\begin{array}{c}0,057 \\
(0,575 \\
(0,055-0,060)\end{array}$ \\
Fatores de segunda ordem & 3026,2 & 1335 & 2,26 & 0,77 & 0,71 & 0,098 & 8,595 \\
Parcela de itens & 563,6 & 119 & 4,96 & 0,86 & 0,76 & $(0,090-0,106)$ & 1,725 \\
\hline
\end{tabular}


Com respeito aos índices de consistência interna [alfa de Cronbach $(\alpha)$, homogeneidade (correlação média interitens, $\mathrm{r}_{\mathrm{m} . \mathrm{i}}$ ) e confiabilidade composta $(C C)$ ] foram computados para cada subfunção, sendo observados os seguintes: experimentação $(\alpha=0,77$, $\left.\mathrm{r}_{\mathrm{m} . \mathrm{i}}=0,27, C C=0,63\right)$, realização $\left(\alpha=0,74, \mathrm{r}_{\mathrm{m} . \mathrm{i}}=0,24\right.$, $C C=0,68)$, existência $\left(\alpha=0,59, \mathrm{r}_{\mathrm{m} . \mathrm{i}}=0,14, C C=0,48\right)$, suprapessoal $\left(\alpha=0,73, \mathrm{r}_{\mathrm{m} . \mathrm{i}}=0,24, C C=0,64\right)$, interativa $\left(\alpha=0,69, \mathrm{r}_{\mathrm{m} . \mathrm{i}}=0,21, C C=0,51\right)$ e normativa $(\alpha=0,76$, $\left.\mathrm{r}_{\mathrm{m} . \mathrm{i}}=0,26, C C=0,75\right)$.

Por fim, testou-se a estrutura proposta pela teoria por meio de escalonamento multidimensional confirmatório, admitindo as especificações previamente listadas. Os índices de ajuste obtidos se mostraram adequados, com Phi de Tucker de 0,97 e Raw Stress de 0,05, indicando a adequação da estrutura bidimensional. O eixo horizontal representa o tipo de orientação enquanto que o vertical representa o tipo de motivador, estando as seis subfunções em regiões diferentes do espaço (Figura 3).

\section{DISCUSSÃO}

O objetivo principal do presente artigo foi conhecer a adequação de um instrumento novo de medida de valores para a teoria funcionalista dos valores (Gouveia, 2003). Os resultados obtidos apontam que a medida apresenta adequada consistência interna e validade de construto aceitável para fins de pesquisa. Ademais, os resultados fornecem suporte empírico para a teoria aqui testada, corroborando plenamente a hipótese de estrutura e parcialmente a de conteúdo. Estes resultados são relevantes pois demonstram a validade da teoria em questão, mesmo partindo de um instrumento diverso do que vem sendo comumente empregado (Gouveia, Milfont et al., 2011; Gouveia et al., 2008), enfocando elementos presumivelmente mais concretos, como são comportamentos descritos.

Neste contexto, partindo do modelo teórico proposto, elaborou-se um instrumento com itens que expressam valores de forma mais concreta, a partir de relatos comportamentais. No caso, providenciou-se uma medida diferente do $Q V B$, sendo mais concreta e contextualizada, requerendo menos abstração ou capacidade cognitiva por parte do respondente. Este instrumento foi elaborado com base em autorrelatos de comportamentos que expressam valores, pois a forma natural de as pessoas seguirem seus valores é se comportando de maneira coerente com eles, de forma a expressá-los ou promovê-los (Bardi \& Schwartz, 2003). Por exemplo, as pessoas que priorizam valores normativos tendem a agir de forma a seguir as normas da sociedade ou a obediência à autoridade, não se envolvendo em comportamentos anticonvencionais. Destaca-se, entretanto, que embora um comportamento possa expressar majoritariamente um determinado valor, certos comportamentos podem ser ambíguos quanto ao valor que expressam, podendo dizer respeito a dois ou mais valores. Por exemplo, o comportamento de fazer trilhas pode expressar

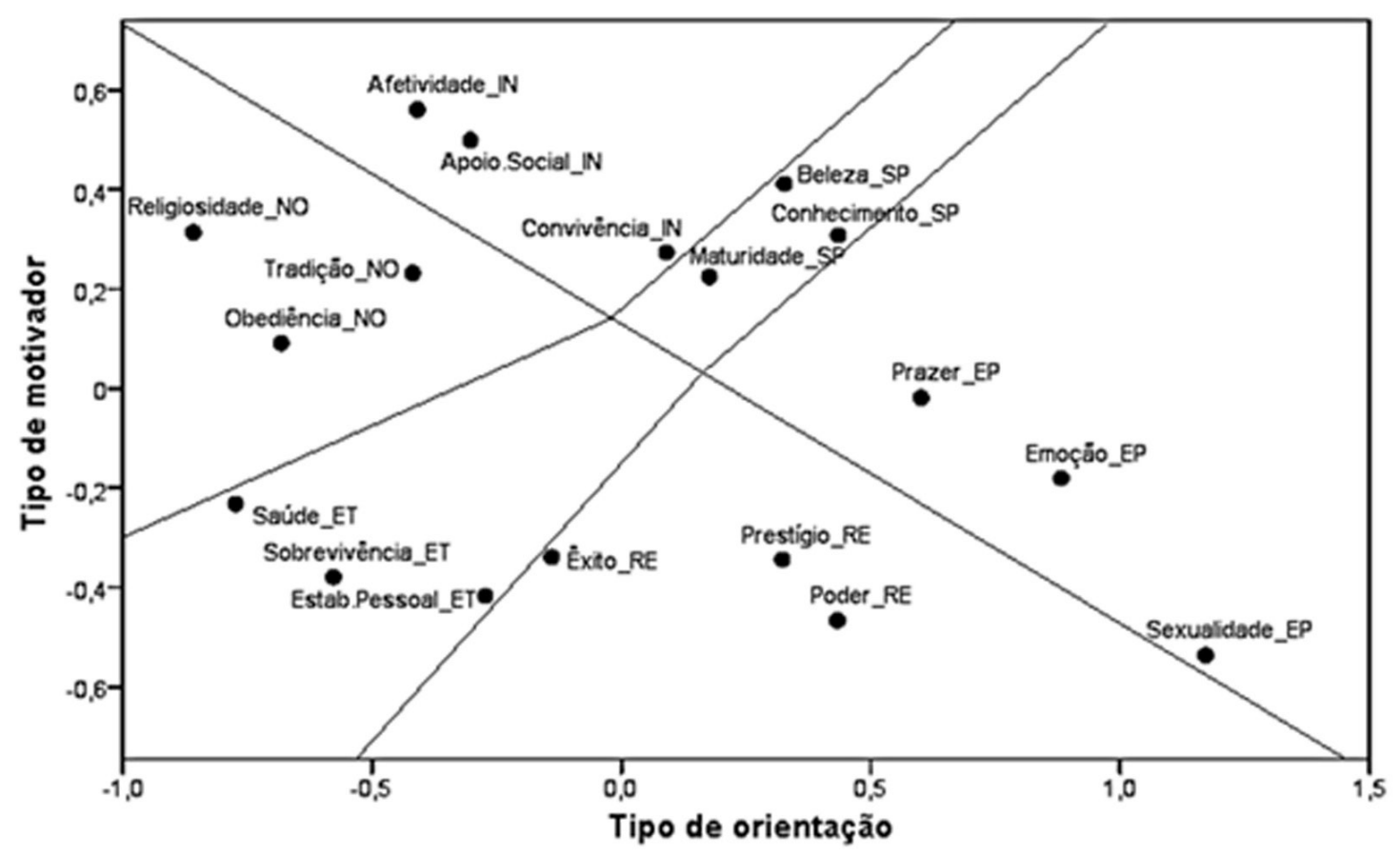

Figura 3. Distribuição espacial dos valores. 
valores de experimentação (busca de aventura) ou suprapessoais (apreciar a beleza da natureza). Não obstante, a elaboração do instrumento selecionou comportamentos que majoritariamente expressam um único valor, excluindo os que apresentassem caráter ambíguo, o que se procurou assegurar a partir da análise dos juízes.

O modelo hexafatorial proposto pela teoria apresentou os melhores índices de ajuste comparativo, estando próximos aos reportados por outros estudos com amostras brasileiras (Gouveia et al., 2009; Medeiros et al., 2012). Embora estes índices tenham se situado próximos ou abaixo do recomendado na literatura (Byrne, 2010), deve-se ter em conta a natureza do construto estudado, já que os valores humanos apresentam pouca variabilidade de resposta, não sendo diferente em outros estudos com a mesma teoria ou com outra, a exemplo da teoria dos tipos motivacionais (Schwartz et al., 2012; Schwartz \& Boehnke, 2004). Em relação à hipótese de estrutura, os resultados apontaram a adequação da estrutura proposta. Em consonância com o pressuposto teórico, existem evidências suficientes para se considerar os valores centrais como localizados entre os pessoais e sociais que, por sua vez, se apresentam em lados opostos do espaço bidimensional, como também a divisão dos valores de acordo com o seu motivador (materialista e idealista).

Os resultados obtidos a partir de três diferentes indicadores de consistência interna são coerentes entre si. A maior parte das subfunções apresentou índices satisfatórios, ou seja, alfa de Cronbach próximo ou acima de 0,70 , índice de homogeneidade acima de 0,20 e confiabilidade composta acima de 0,60. Apenas a subfunção existência obteve índices abaixo do esperado. Embora esta tenha apresentado um valor de $\mathrm{CC}$ de 0,48 , os outros dois índices (alfa de 0,59 e homogeneidade de 0,14 ) foram próximos ao aceitável na literatura para fins de pesquisa, ou seja, alfa e homogeneidade próximos de 0,60 e 0,15, respectivamente (Garson, 2013). Ademais, o alfa de Cronbach, índice mais amplamente empregado na literatura, tende a ser um estimador conservador da fidedignidade de uma medida, pelo que, a verdadeira estimativa de fidedignidade tem baixa probabilidade de ser menor e elevada probabilidade de ser maior do que o valor reportado (Maroco \& Garcia-Marques, 2006). Além disso, as características do construto e da amostra empregadas também podem afetá-lo, pois fenômenos que apresentam pouca variabilidade de resposta inter-sujeitos, a exemplo dos valores humanos, tendem a apresentar valores abaixo dos comumente recomendados (Rodriguez \& Maeda, 2006).
Em conclusão, a medida ora proposta apresenta parâmetros de validade e precisão adequados para justificar sua utilização em estudos futuros. Uma vez que a subfunção existência apresentou limitações quanto à consistência interna, estudos futuros que empreguem esta medida poderão reformular ou substituir os itens relacionados a esta com o intuito de melhorar sua precisão. Isso é possível em razão de a validade do conteúdo da teoria não ser condicionada a um conjunto de valores/itens específicos, mas sim a representação adequada das seis subfunções. De modo geral, os resultados obtidos demonstram evidências que reforçam a validade da teoria funcionalista dos valores, que se mostrou independente do método de mensuração empregado. Por fim, estudos futuros poderão fornecer evidências de validade convergente e discriminante do $Q V B-R C$, tendo em conta sua relação com outras variáveis, tais como atitudes (religiosa, ambiental), traços de personalidade e variáveis pessoais (idade, educação, sexo) ou, inclusive, com respeito ao $Q V B$.

\section{REFERÊNCIAS}

Ardila, R., Gouveia, V.V., \& Medeiros, E.D. (2012). Human values of colombian people. Evidence for the functionalist theory of values. Revista Latinoamericana de Psicologia, 44, 105-117.

Bardi, A., \& Schwartz, S. H. (2003). Values and behavior: Strength and structure of relations. Personality and Social Psychology Bulletin, 29, 1207-1220. doi: 10.1177/0146167203254602

Borg, I., Groenen, P. J. F., \& Mair, P. (2012). Applied Multidimensional Scaling. New York: Springer.

Byrne, B. M. (2010). Structural equation modeling with Amos: Basic concepts, applications, and programmimg ( $2^{\mathrm{a}}$ ed.). New York: Routledge.

Fischer, R., Milfont, T. L., \& Gouveia, V. V. (2011). Does Social Context Affect Value Structures? Testing the Within-Country Stability of Value Structures With a Functional Theory of Values. Journal of Cross-Cultural Psychology, 42, 253-270. doi: $10.1177 / 0022022110396888$

Garson, G. D. (2013). Scales and Measures. Asheboro: Statistical Associates Publishers.

Garson, G. D. (2012). Structural Equation Modeling. Asheboro: Statistical Publishing Associates.

Gouveia, V. V. (2003). A natureza motivacional dos valores humanos: Evidências acerca de uma nova tipologia. Estudos de Psicologia (Natal), 8, 431-443.

Gouveia, V. V. (2013). Teoria funcionalista dos valores humanos. Fundamentos, aplicações e perspectivas. São Paulo: Casa do Psicólogo.

Gouveia, V. V., Fonseca, P. N., Milfont, T. L., \& Fischer, R. (2011). Valores humanos: Contribuições e perspectivas teóricas. In C. V. Torres \& E. R. Neiva (Eds.). A psicologia social: Principais temas e vertentes (pp. 296-313). Porto Alegre, RS: ArtMed.

Gouveia, V. V., Milfont, T. L., Fischer, R., \& Coelho, J. A. P. M. (2009). Teoria funcionalista dos valores humanos: Aplicações para organizações. Revista de Administração Mackenzie, 10 , 34-59. doi: 10.1590/S1678-69712009000300004 
Gouveia, V. V., Milfont, T. L., Fischer, R., \& Santos, W. S. (2008). Teoria funcionalista dos valores humanos. In M. L. M. Teixeira (Ed.). Valores humanos e gestão: Novas perspectivas (pp. 47-80). São Paulo, SP: Senac.

Gouveia, V. V., Milfont, T. L., Soares, A. K. S., Andrade, P. R., \& Leite, I. L. (2011). Conhecendo os valores na infância: Evidências psicométricas de uma medida. Psico, 42, 106-115.

Gouveia, V. V., Santos, W. S., Milfont, T. L., Fischer, R., Clemente, M., \& Espinosa, P. (2010). Teoría funcionalista de los valores humanos en España: Comprobación de las hipótesis de contenido y estructura. Revista Interamericana de Psicologia, 44, 203-214.

Hair Jr., J. F., Black, W. C., Babin, B. J., \& Anderson, R. E. (2009). Multivariate Data Analysis (7th ed.). Upper Saddle River: Prentice Hall.

Inglehart, R. (1977). The silent revolution: Changing values and political styles among Western publics. Princeton, NJ: Princeton University Press.

Inglehart, R. (1989). Cultural shift in advanced industrial society. Princeton, NJ: Princeton University Press.

Little, T. D., Cunningham, W., Shahar, G., \& Widaman, K. F. (2002). To parcel or not to parcel: Exploring the question, weighing the merits. Structural Equation Modeling, 9, 151-173. doi: 10.1207/S15328007SEM0902 1

Kluckhohn, C. (1951). Values and value orientations in the theory of action. In T. Parsons, \& E. Shils (Eds.). Toward a general theory of action (pp. 388-433). Cambridge, MA: Harvard University Press.

Maio, G. R. (2010). Mental representations of social values. In M. P. Zanna (Ed.), Advances in experimental social psychology (Vol. 42; pp. 1-43). New York: Academic Press.

Maslow, A. H. (1954). Motivation and personality. New York, NY: Harper and Row.

Marroco, J. \& Garcia-Marques, T. (2006). Qual a fiabilidade do alfa de Cronbach? Questões antigas e soluções modernas. Laboratório de Psicologia, 4, 65-90.

Medeiros, E. D., Gouveia, V.V., Gusmão, E. E. S., Milfont, T. L., Fonsêca, P. N., \& Aquino. T. A. A. (2012). Teoria funcionalista dos valores humanos: Evidências de sua adequação no contexto paraibano. RAM. Revista de Administração Mackenzie, 13, 18-44. doi: 10.1590/S1678-69712012000300003

Parsons, T. \& Shils, E. A. (1951). Toward a general theory of action. New York, NY: Harper.

Rodriguez, C. R., \& Maeda, Y. (2006). Meta-analysis of coefficient alpha. Psychological Methods, 11, 306-322. doi: 10.1037/1082989X.11.3.306
Rokeach, M. (1973). The nature of human values. New York, NY: Free Press.

Ronen, S. (1994). An underlying structure of motivational need taxonomies: A cross-cultural confirmation. In H. C. Triandis, M. D. Dunnette, \& L. M. Hough (Eds.). Handbook of industrial and organizational psychology (Vol. 4; pp. 241-269). Palo Alto, CA: Consulting Psychologists Press.

Ros, M. \& Gouveia, V.V. (2006). Psicologia social dos valores humanos: Desenvolvimentos teóricos, metodológicos e aplicados. São Paulo: Editora Senac.

Schwartz, S. H. (1992). Universal in the content and structure of values: Theoretical advances and empirical tests in 20 countries. In M. P. Zanna (Ed.). Advances in experimental social psychology (pp. 1-65). New York, NY: Academic Press.

Schwartz, S. H., \& Boehnke, K. (2004). Evaluating the structure of human values with confirmatory factor analysis. Journal of Research in Personality, 38, 230-255. doi: 10.1016/S00926566(03)00069-2

Schwartz, S.H., Cieciuch, J., Vecchionem M., Davidov, E., Fischer, R., Beierlein, C., et al. (2012). Refining the Theory of Basic Individual Values. Personality processes and individual differences, 103, 663-668. doi: 10.1037/a0029393

Schwartz, S. H., Melech, G., Lehmann, A., Burgess, S., Harris, M., \& Owens, V. (2001). Extending the cross-cultural validity of the theory of basic human values with a different method of measurement. Journal of Cross-Cultural Psychology, 32, 519-542. doi: 10.1177/0022022101032005001

Thomas, W. I. \& Znaniecki, F. (1918). The Polish peasant in Europe and America. Boston, MA: University of Chicago Press.

Verplaken, B. \& Holland, R. W. (2002). Motivated decision making: Effects of activation and self-centrality of values on choices and behavior. Journal of Personality and Social Psychology, 82, 434-447. doi: 10.1037/0022-3514.82.3.434

Autores:

Tiago Jessé Souza de Lima - Doutorando, Universidade Federal da Paraíba. Valdiney Veloso Gouveia - Doutor, Universidade Federal da Paraíba. Luana Elayne Cunha de Souza - Doutora, Universidade Federal da Paraíba. Patrícia Nunes Fonseca - Doutora, Universidade Federal da Paraíba.

Endereço para correspondência:

Tiago Jessé Souza de Lima

Rua Lindolfo Gonçalves Chaves, 513, apto. 202

CEP 58051-200 João Pessoa, PB, Brasil

E-mail: tiago.souzalima@hotmail.com

Recebido em: 12.11 .13

Aceito em: 07.01.14 